\title{
Raciocínio Lógico e Escolarização
}

\section{Maria da Graça B. B. Dias \\ Antonio Roazzi}

Universidade Federal de Pernambuco (UFPE)

O objetivo deste estudo foi investigar o efeito do modo defaz-deconta, formas de silogismos e tipos de fatos, no desempenho de crianças inglesas de 5 anos de idade, escolarizadas, de nivel sócio-econômico (NSE) médio e de crianças brasileiras de 5 anos de idade, nao escolarizadas de NSE baixo. Os resultados mostraram que as crianças, independentemente se escolarizadas ou nao, produziram maior número de respostas corretas e maior número de justificativas teóricas na condição de brincadeira de faz-de-conta do que na condição verbal comum. Este efeito foi especialmente marcante para os fatos desconhecidos e para osfatos contrários às experiências das crianças. Este padrão foi mantido para ambasformas de silogismos, embora em geral o desempenho das crianças nos problemas envolvendo Modus Ponens tenha sido mais acurada do que nos problemas envolvendo Modus Tollens. As crianças não-escolarizadas obtiveram menores escores do que as escolarizadas. No entanto, esta diferença não foi tão forte como aquela relatada por Scribner (1977) onde as crianças não-escolarizadas obtiveram baixos escores adotando um viés empírico. Em nosso estudo, apesar das crianças não-escolarizadas terem freqüentemente recorrido a justificativas arbitrárias, puderam recorrer a uma atitude teórica quando a condição defaz-de-conta foi adotada.

\section{Introdução}

O estudo investiga o efeito da escolarização no desenvolvimento intelectual através de tarefas que requerem raciocínio lógico. Em particular, tenta-se verificar se o fato de a criança freqüentar a escola afeta seu 
desempenho em tarefas de raciocínio lógico verbal apresentadas sob a forma de silogismos. Por silogismo entende-se um problema lógico apresentado através de um conjunto de premissas gerais e de uma conclusão baseada nessas premissas.

O estudo de Luria (1976), conduzido no Ubequistão na Ásia Central, nos anos de 1931 e 1932, representa uma das primeiras tentativas de pesquisa sobre este tema. $\mathrm{O}$ interesse de Luria não era dirigido somente para os efeitos da escolarização sobre os processos mentais mas também para as mudanças acarretadas pela Revolução Russa e pela introdução de novas tecnologias. Para Luria, os sujeitos analfabetos e de comunidades tradicionais manifestariam menos habilidade em lidar com diversos tipos de problemas, inclusive com problemas silogísticos, do que aqueles que tiveram instrução formal ou que estivessem integrados $\mathrm{cm}$ comunidades de organização mais coletiva. Para testar sua hipótese Luria apresentou aos diferentes grupos de sujeitos silogismos cujas premissas continham dados familiares, pois referiam-se à experiência prática dos sujeitos, ou dados não familiares que requeriam respostas baseadas em inferências puramente teóricas. Os resultados desse estudo mostram que os sujeitos analfabetos resolveram $60 \%$ dos problemas silogísticos envolvendo conteúdos familiares. No entanto, o desempenho desses sujeitos decaiu para $15 \%$ quando o conteúdo era desconhecido. Já o grupo composto por sujeitos que tinham alguma instrução, ou que estavam habituados a formas de comunicação mais complexas, obteve $100 \%$ de acertos em ambos os tipos de silogismos, aceitando as premissas como base para seu raciocínio e compreendendo a universalidade das mesmas.

Mais recentemente outros estudos focalizaram a relação entre escolarização e raciocínio lógico. Expoentes neste campo são os trabalhos de Cole, Gay, Glick e Sharp (1971), Scribner (1975) com a população kpelle na África Ocidental e o de Sharp e Cole (citado por Scribner, 1977) entre os maias do Yucatan, no México. Em geral, estes estudos mostram resultados surpreendentemente uniformes e apontam para uma mesma direção: 
Cole, Gay, Glick e Sharp (1971) utilizaram vários tipos de problemas lógicos verbais em contextos naturalista e experimental, com o objetivo de estudar os processos inferenciais entre crianças e adultos kpelles. Em um destes estudos, com crianças de 10 a 14 anos de idade com diversos graus de escolarização, foram utilizados problemas como o seguinte:

Se Flumo ou Yakpalo bebe aguardente, o chefe da cidade fica zangado.

Flumo não está bebendo aguardente. Yakpalo está bebendo aguardente.

O chefe da cidade está zangado?

As crianças que nunca freqüentaram a escola deram apenas 30\% de respostas corretas. Em contraste, as crianças que estavam no terceiro grau solucionaram $90 \%$ dos problemas. Para os autores, a fonte aparente do desempenho "não lógico" da população não escolarizada parece ser o fracasso em integrar e reter as informações contidas nas premissas dos problemas.

Os objetivos dos estudos exploratórios de Scribner (1975) eram verificar se a lógica estaria relacionada com a cultura e identificar os processos envolvidos no raciocínio inferência! dentro de uma cultura.

Em um primeiro estudo fizeram parte sujeitos analfabetos kpelles, membros de uma tribo situada em região isolada da África Ocidental; sujeitos desta mesmo tribo mas que tinham tido educação secundária, e um terceiro grupo formado por estudantes norte-americanos. Cada um dos sujeitos recebeu problemas silogísticos com conteúdos envolvendo fatos verdadeiros, ou fatos não verídicos.

Os sujeitos analfabetos alcançaram 53\% de acertos em ambos os problemas, os estudantes kpelles obtiveram $80 \%$ e os universitários norteamericanos $90 \%$ de respostas corretas. Não foram apresentados dados sobre possíveis diferenças nas respostas aos silogismos envolvendo fatos verídicos comparados aos não verídicos.

Neste estudo, Scribner analisou as justificativas oferecidas pelos sujeitos às suas respostas. Três tipos de justificativas foram classificadas: Teóricas, quando os sujeitos referiam-se às premissas do problema; Empíricas, quando levavam em consideração o próprio conhecimento ou 
acreditavam ser a verdade; e Arbitrário, quando os mesmos não justificavam ou eram explicações irrelevantes.

Com esta análise, os resultados mostram que os analfabetos kpclles ofereceram $23.3 \%$ de justificativas teóricas, $68.1 \%$ de empíricas e $9.6 \%$ de arbitrárias. $\mathrm{O}$ grupo de estudantes kpelles alcançou $75 \%$ de justificativas teóricas, $21.9 \%$ de empíricas e $3.1 \%$ de arbitrárias. Já os universitários norte-americanos obtiveram $82.3 \%$ de explicações teóricas, 3.1\%) de empíricas e $14.6 \%$ de arbitrárias.

Pode-se notar que as justificativas teóricas foram mais utilizadas pelos kpelles escolarizados e pelos universitários norte-americanos, enquanto os sujeitos analfabetos recorreram $\mathrm{cm}$ maior número às empíricas.

Scribner levanta a possibilidade de esta discrepância entre o número de erros e o tipo de justificativas dos analfabetos c estudantes ser devida à inclusão de afirmações de verdade do tipo "Eu sei", "Eu acho", "Eu acredito", encontradas apenas entre os analfabetos que transformavam o problema hipotético $\mathrm{cm}$ factual.

Resultados comparáveis aos de Scribner (1975), Luria (1976) c de Cole, Gay, Glick c Sharp (1971) foram obtidos por Sharp e outros (citados por Scribner, 1977). Eles replicaram os estudos dos kpelles entre crianças c adultos escolarizados c analfabetos das zonas rurais c semi-rurais de Yucatan no México. Resultados similares também foram encontrados por Cole c Scribner (1974) com uma amostra de 750 vais.

Revendo estes estudos, Scribner (1977, p.485) concluiu que "não somente os resultados quantitativos são extremamente uniformes de estudo para estudo, como também certos aspectos qualitativos do desempenho são tão similares que se toma frequientemente difícil distinguir o protocolo de um sujeito do Ubequistão de um vai - não obstante a distância cultural e geográfica". Estes estudos transculturais também demonstram uma diferença significativa entre o número de respostas corretas entre os sujeitos escolarizados e não escolarizados dentro de uma mesma cultura. Assim, para Scribner o nível educacional, mais do que a cultura, exerce um papel efetivo no desempenho dos sujeitos.

Em seus estudos comparativos, Scribner mostra também a grande diferença entre os sujeitos escolarizados c não escolarizados com referência ao número de justificativas teóricas para as respostas. $\mathrm{O}$ viés empírico foi 
freqüentemente encontrado entre os sujeitos não escolarizados, enquanto os sujeitos escolarizados adotavam a atitude teórica - "mesmo crianças de 7 anos de idade, da segunda série de sistemas escolares conhecidos por enfatizarem um aprendizado mecânico $\mathrm{cm}$ lugar do desenvolvimento do pensamento crítico, tendem a se referir ao que o problema relata quando são questionados sobre suas respostas" (Scribner, 1977, p.489). Estes resultados sugerem duas conclusões: primeiro, que apenas um pouco de escolarização é suficiente para produzir um aumento substancial no desempenho dos sujeitos e, segundo, que a escolarização é uma condição necessária para um desempenho acima da média, pelo menos para os silogismos onde os sujeitos não podem contar com seus conhecimentos práticos.

No entanto, Hawkins, Pca, Glick e Scribner (1984), em estudo com pré-escolares c crianças escolarizadas, constataram que ambos os grupos de sujeitos obtiveram bons resultados nos problemas silogísticos com conteúdos desconhecidos do tipo fantasia ou com conteúdos congruentes com as experiências das crianças. Nos problemas com conteúdos incongruentes, tanto as crianças escolarizadas como as não escolarizadas alcançaram resultados abaixo da média. Os dados também mostram que as crianças davam um número significativamente maior de respostas corretas c justificativas teóricas na condição $\mathrm{cm}$ que recebiam os problemas envolvendo fantasia em primeiro lugar, do que nas outras condições onde este tipo de conteúdo era apresentado $\mathrm{cm}$ segundo ou terceiro lugar.

Resultados semelhantes foram encontrados por Dias c Harris (1988a) onde crianças do pré-escolar apresentaram bom desempenho $\mathrm{cm}$ problemas silogísticos, mesmo quando o conteúdo era contrário às suas experiências, desde que a tarefa fosse apresentada $\mathrm{cm}$ um contexto de brincadeira de faz-de-conta. Para os autores, as crianças parecem construir um mundo de faz-de-conta no qual as premissas são aceitas mesmo sem o respaldo de suas experiências diárias. Assim, pode-se notar que, sob certas circunstâncias, crianças com limitada exposição à escolarização podem raciocinar de maneira lógica c teórica.

O objetivo deste estudo foi investigar o efeito do modo de brincadeira de faz-de-conta, tipo de conteúdo envolvido nas premissas c forma de silogismo $\mathrm{cm}$ crianças escolarizadas de nível sócio-econômico médio 
(NSE) da Inglaterra e do Brasil, e crianças analfabetas de NSE baixo do Brasil.

Neste estudo os conteúdos utilizados nos problemas incluíam fatos conhecidos e coerentes com as experiências empíricas das crianças, outros contrários a essas experiências e fatos por elas desconhecidos.

Outra variável independente utilizada foi a forma do silogismo. Dois tipos de inferências válidas, Modus Ponens (p implica q, p, portanto q) e Modus Tollens ( $\mathrm{p}$ implica q, não q, portanto não p); foram incluídas a fim de estender os resultados anteriormente encontrados por Dias e Harris (1989) com crianças escolarizadas. Este estudo demonstrou que apesar de os problemas envolvendo Modus Tollens tenham provado serem mais difíceis que os que envolviam Modus Ponens, o modo de apresentação (contexto de brincadeira de faz-de-conta) ajuda as crianças a lidarem melhor com as duas formas de silogismo. Assim, procuramos aqui comparar o desempenho de crianças analfabetas e de crianças escolarizadas nestas duas formas de silogismo.

\section{Método}

SUJEITOS

Fizeram parte do estudo 144 crianças variando de 5 anos e 0 meses de idade a 5 anos e 11 meses (média de 5 anos e meio). Dessas crianças 48 eram inglesas de NSE médio, que freqüentavam classes de alfabetização de duas escolas do $1^{\circ}$ grau (Primary School) da cidade de Oxford, Inglaterra; 48 crianças brasileiras de NSE médio, que freqüentavam classes de alfabetização de duas escolas do $1^{\circ}$ grau da cidade do Recife; e 48 crianças também brasileiras da cidade do Recife, porém de NSE baixo e que nunca freqüentaram escolas.

MATERIAL

O material consistiu de 24 problemas silogísticos em forma de Modus Ponens e 24 problemas em forma de Modus Tollens utilizados em Dias e Harris (1988a e 1989; ver Tabela 1 para alguns exemplos). Para cada 
forma de problema, os conteúdos das premissas eram de três tipos diferentes: oito incluíam fatos conhecidos que concordavam com a experiência das crianças, oito incluíam fatos desconhecidos e oito incluíam fatos contrários à experiência do dia-a-dia. Perguntas de sondagem (ver Tabela 1 para exemplo) foram utilizadas a fim de estabelecer que fatos as crianças realmente sabiam.

Tabela 1 - Perguntas de sondagem

Fatos Conhecidos
Modus
Ponens
Modus, Tollens

(Pergunta de Sondagem: Que barulho os gaios fazem?)

Todos os gatos miam;

Tot é um gato;

Ele mia?
Tot

Tot
Todos os gatos miam; só sabe latir; é diferente de um gato?

Fatos Desconhecidos

(Pergunta de Sondagem: Que barulho as hienas fazem?)

Todas as hienas riem;

Tot é uma hiena;

Ela ri?
Todas hienas riem;

Tot só sabe chorar;

Ela é diferente de uma hiena?

\section{Fatos Contrários}

(Pergunta de Sondagem: Que baralho os gatos fazem?)

Todos os gatos latem;

Tot é um gato;

Ele late?
Todos os gatos latem;

Tot só sabe miar;

Ele é diferente de um gato?

PROCEDIMENTO

Metade das crianças de cada amostra, se brasileira ou inglesa, recebeu os problemas na forma de Modus Ponens e a outra metade na forma de 
Modus Tollens. Dentro de cada um desses subgrupos, metade das crianças pertenciam ao Grupo Verbal c metade ao Grupo de Brincadeira. Cada subgrupo de 12 crianças foi randomicamente dividido $\mathrm{cm}$ três subgrupos, c a cada subgrupo de quatro crianças foi apresentado um conjunto de oito problemas da mesma forma, com conteúdos que eram ou conhecidos, ou desconhecidos, ou contrários às experiencias das crianças. A ordem dos oito problemas foi randomicamente variada pelos sujeitos.

Antes de iniciar a tarefa, as perguntas de sondagem foram apresentadas, a fim de determinar se as crianças eram ou não familiarizadas com os fatos contidos na primeira premissa de cada problema.

Para o Grupo Verbal as instruções foram as seguintes: "Eu vou ler para vocês algumas historinhas sobre coisas que: a) você conhece (para o subgrupo de Fatos Conhecidos); b) você não conhece (para o subgrupo de Fatos Desconhecidos), e c) você achará estranhas (para o subgrupo de Fatos Contrários). Porem, vamos fazer de conta que todas as coisas das estórias são verdadeiras." O experimentador então lia cada problema com voz normal.

Para as crianças do Grupo de Brincadeira as instruções foram as seguintes: "Vamos fazer de conta que estou $\mathrm{cm}$ outro planeta. Tudo neste planeta a) você conhece (para o subgrupo de Fatos Conhecidos); b) você não conhece (para o subgrupo de Fatos Desconhecidos), c c) você achará estranhas (para o subgrupo de Fatos Contrários)." O experimentador apresentava cada problema como uma estória, isto é, sua entonação era a de uma maneira de faz-de-conta.

Antes de responder à questão de cada silogismo, as crianças tinham de mencionar a primeira e a segunda premissas de cada silogismo na ordem dada, a fim de nos certificarmos se elas haviam registrado o problema. Se algum erro ocorria, o problema era repetido ate no máximo três vezes. Depois, a pergunta era feita, $\mathrm{c}$ finalmente o experimentador pedia à criança para justificar a resposta dada: "Por que você respondeu sim?" ou "Por que você respondeu não?" Dependendo da resposta outra pergunta era feita: "Como você sabe disso?" Esta última questão era colocada apenas quando a resposta da criança não era clara, isto c, quando a resposta não especificava de onde vinha o conhecimento da criança. 


\section{Resultados}

Todas as respostas foram classificadas de duas formas: a) por nùmero de respostas corretas, e b) por tipos de justificativas dadas às respostas, independentemente de serem certas ou erradas. As justificativas foram classificadas como teóricas, empíricas ou arbitrárias, seguindo os mesmos critérios de Scribner (1975) e Dias (1987).

Justificativas teóricas eram as explicações que se referiam somente às informações presentes na primeira, segunda ou em ambas as premissas de maneira dedutivamente válida. Justificativas empíricas eram aquelas que se referiam ao conhecimento prático do mundo. Já as justificativas arbitrárias eram as explicações irrelevantes, ou quando a criança não oferecia nenhuma justificativa.

Antes de responder aos problemas silogísticos, as crianças receberam oito questões de sondagem. Os dados indicam que as crianças eram familiarizadas com os princípios gerais presentes nos problemas com conteúdo conhecido, não familiarizadas com aqueles de conteúdo desconhecidos $\mathbf{c}$ mantinham opiniões contrárias àquelas presentes nos problemas com fatos contrários (ver Tabela 2).

\section{Tabela 2 - Percentagem de respostas corretas nas Perguntas de Sondagem em função da Idade e Tipo de Fato nas três amostras}

Amostra

Conhecido

$98.4 \%$

$96.9 \%$

$95.3 \%$
Tipo de Fato

Desconhecido

Contrário

Inglesas

Brasileiras

-Escolarizadas

- Não escolarizadas

$0.78 \%$

$100.00 \%$
$3.10 \%$

$0.00 \%$
$96.1 \%$

$93.70 \%$ 
A Tabela 3 mostra que, em termos gerais, o Grupo de Brincadeira obteve maior número de respostas corretas do que o Grupo Verbal, principalmente nos Fatos Desconhecidos e nos Fatos Contrários. Nota-se que o desempenho das crianças nos problemas envolvendo Modus Ponens foi um pouco maior do que nos problemas envolvendo Modus Tollens, e que para as crianças não escolarizadas as médias de acerto foram menor do que as médias alcançadas pelas crianças escolarizadas independente da nacionalidade.

Tabela 3 - Média de respostas corretas (de um total de 8) em função de Grupo, Tipo de Fato e Forma de Silogismo nas três amostras

\begin{tabular}{|c|c|c|c|c|c|c|c|}
\hline \multirow{3}{*}{$\begin{array}{l}\text { Amostra } \\
\text { Inglesas }\end{array}$} & \multirow{2}{*}{$\begin{array}{l}\text { Forma } \\
\text { MP }\end{array}$} & \multicolumn{3}{|c|}{$\begin{array}{l}\text { Grupo Verbal } \\
\text { Conhec.Desc.Contr. }\end{array}$} & \multicolumn{3}{|c|}{$\begin{array}{l}\text { Grupo de Brinc. } \\
\text { Conhec.Desc.Contr. }\end{array}$} \\
\hline & & 8.00 & 6.00 & 4.75 & 8.00 & 7.50 & 8.00 \\
\hline & MT & 7.75 & 4.00 & 2.75 & 8.00 & 7.75 & 7.75 \\
\hline & Médias & 7.87 & 5.00 & 3.75 & 8.00 & 7.62 & 7.87 \\
\hline \multirow{2}{*}{$\begin{array}{l}\text { Brasileiras } \\
\text {-Escolarizadas }\end{array}$} & MP & 7.75 & 6.75 & 2.75 & 7.50 & 6.75 & 7.50 \\
\hline & MT & 7.75 & 6.25 & 3.50 & 8.00 & 7.25 & 6.25 \\
\hline \multirow{4}{*}{$\begin{array}{l}\text { Brasileiras } \\
\text {-Não Escolarizadas }\end{array}$} & Médias & 7.75 & 6.50 & 3.12 & 7.75 & 7.00 & 6.87 \\
\hline & MP & 6.50 & 4.50 & 2.75 & 7.75 & 6.75 & 7.75 \\
\hline & MT & 7.25 & 3.50 & 2.25 & 7.00 & 6.25 & 6.00 \\
\hline & Médias & 6.87 & 4.00 & 2.50 & 7.37 & 6.50 & 6.87 \\
\hline
\end{tabular}


Com o objetivo de checar estas conclusões, foi feita uma análise de variância de Grupo (2), Tipos de Fato (3), Formas de Silogismo (2) e Amostra (3), como fatores e número de acertos como variável dependente. Esta análise produziu um efeito significativo para Grupo $(\mathrm{F}(1,108)=$ 78.56, $\mathrm{p}<.001)$, para Tipos de Fato $(\mathrm{F}(2,108)=85.18$, $\mathrm{p}<.001)$, para Formas de Silogismo $(\mathrm{F}(1,108)=8.35, \mathrm{p}<.01)$ e para Amostra $(\mathrm{F}(2,108)=$ $64.85, \mathrm{p}<.001)$. Esta análise mostrou, ainda, as seguintes interações: Grupo e Tipos de Fato $(\mathrm{F}(2,108)=53.23, \mathrm{p}<.001)$, Amostra e Grupo $(\mathrm{F}(2,108)=$ 4.41, $\mathrm{p}<.01)$ e Amostra c Tipos de Fato $(\mathrm{F}(4,108)=2.49$, $\mathrm{p}<.05)$.

$\mathrm{O}$ efeito significativo para grupo, maior número de respostas corretas no contexto de faz-de-conta (média 7.32) do que no contexto verbal (média .26), foi mostrado diversas vezes (Dias e Harris, 1988a, 1989 e 1990; Holanda, 1989; Santos, 1989 etc). Já a melhor performance nos silogismos envolvendo Modus Ponens (média 6.51) do que naqueles envolvendo a forma Modus Tollens (média 6.07), foi mostrado por Braine e Rumain 1983), Dias e Harris (1988b).

A média de respostas corretas nas três amostras foram comparadas usando o Teste de Newman-Keuls. Esta análise mostrou que as crianças escolarizadas, tanto inglesas como brasileiras (médias respectivas 6.69 e 6.50), ofereceram significativamente maior número de respostas corretas do que as crianças não escolarizadas (média 5.69, p<.01). Isto mostra a nfluência da escolarização no desempenho em problemas silogísticos.

Os três tipos de fatos foram também analisados através do mesmo teste estatístico. Como nos estudos de Dias e Harris (1988a), os fatos conhecidos (média 7.60) obtiveram um número significativamente maior de acertos do que os desconhecidos (média 6.10, p<.01) e contrários média 5.17, p<.01). Por sua vez, os fatos desconhecidos obtiveram significativamente maior número de respostas corretas do que os fatos contrários $(\mathrm{p}<.01)$.

Este mesmo teste foi aplicado a fim de se analisar a interação entre Amostra e Grupo. No Grupo Verbal as crianças não escolarizadas 
ofereceram menor número de acertos que as crianças inglesas $(\mathrm{p}<.01) \mathrm{e}$ de que as brasileiras escolarizadas $(\mathrm{p}<.01)$, enquanto estas duas amostras de crianças escolarizadas não diferiram significativamente no número de respostas corretas. Já no Grupo de Brincadeira, apesar de as crianças inglesas ofereceram maior número de acertos do que as brasileiras não escolarizadas, esta diferença nao alcançou nível de significância. Não houve, também, diferença significativa entre as crianças escolarizadas inglesas e brasileiras, como também entre as brasileiras tanto escolarizadas como não escolarizadas. Esta análise mostrou ainda que entre todas as três amostras o número de respostas aumentou significativamente entre o Grupo verbal e o Grupo de Brincadeira $(\mathrm{p}<.01)$. A amostra que mais se beneficiou do contexto de brincadeira foi a de crianças não escolarizadas (aumento médio 2.46), seguida da amostra de crianças inglesas (aumento médio 2.29) e por último pela amostra de crianças brasileiras escolarizadas (a média 1.42). Estes resultados vêm corroborar, uma vez mais, o efeito da brincadeira de faz-de-conta no número de acertos quando crianças resolvem silogismos (Dias c Harris, 1988a, 1989 e 1990).

A análise com o teste de Newman-Keuls para a interação entre Amostra e Tipos de Fatos mostrou que entre as crianças inglesas foi oferecido maior número de respostas corretas para os fatos conhecidos do que para os contrários $(\mathrm{p}<.01)$ e os desconhecidos $(\mathrm{p}<.01)$. O número de acertos para os fatos contrários e desconhecidos não diferiu significativamente. Entre as crianças brasileiras escolarizadas, o número de acertos dados aos fatos conhecidos foi significativamente maior que aqueles oferecidos aos fatos contrários $(\mathrm{p}<.01)$ e desconhecidos $(\mathrm{p}<.01)$. Essas crianças ofereceram também maior número de respostas corretas aos problemas envolvendo fatos desconhecidos, quando comparados aos fatos contrários. Já as crianças brasileiras não escolarizadas, obtiveram maior número de acertos nos fatos conhecidos quando comparados aos fatos contrários $(\mathrm{p}<.01)$ e desconhecidos $(\mathrm{p}<.01)$. O número de respostas corretas oferecido aos fatos desconhecidos não difere significativamente daquele oferecido aos fatos contrários. 
A análise com o Teste de Newman-Keuls revelou também que para os fatos conhecidos o número de acertos das três amostras não difere significativamente. No entanto o número de acertos dados aos fatos desconhecidos pelas crianças escolarizadas inglesas e brasileiras foi significativamente maior que o número de acertos oferecidos pelas crianças não escolarizadas $(\mathrm{p}<.01)$. Já nos fatos contrários, as crianças inglesas ofereceram maior número de respostas corretas do que as brasileiras não escolarizadas $(\mathrm{p}<.01)$ e as escolarizadas $(\mathrm{p}<.05)$. Os grupos de crianças brasileiras não diferiram significativamente neste tipo de conteúdo. Este efeito interativo significativo mostra claramente que à medida que os conteúdos dos silogismos aumentam em nivel de dificuldade o grau de escolarização passa a exercer um papel mais relevante.

A média de respostas corretas nos dois grupos nos três tipos de 'atos foram comparados usando-se o Teste de Newman-Keuls, para se analisar a interação entre Grupo e Tipos de Fatos. Esta análise mostrou que no Grupo Verbal um número significativamente maior de respostas corretas foi oferecido para os fatos conhecidos, quando comparados com os fatos contrários $(\mathrm{p}<.01)$ e com os fatos desconhecidos $(\mathrm{p}<.01)$. $\mathrm{O}$ número de respostas corretas para estes dois últimos tipos de conteúdo diferiu significativamente $(\mathrm{p}<.01)$. No Grupo de Brincadeira todas as comparações possíveis foram não significativas.

Esta análise mostrou também que o número de acertos oferecidos aos fatos conhecidos não diferiu significativamente entre os dois grupos. ${ }^{\wedge}$ los fatos desconhecidos, o número de acertos foi significativamente maior no Grupo de Brincadeira quando comparado ao Grupo Verbal ( $\mathrm{p}<.01)$. O mesmo ocorreu nos fatos contrários, onde as crianças pertencentes ao Grupo de Brincadeira ofereceram maior número de respostas corretas do que aquelas do Grupo Verbal ( $\mathrm{p}<.01)$. Estes resultados provenientes da interação Grupo X Tipo de Fato foram semelhantes aos encontrados em Dias e Harris (1988a), onde o efeito da brincadeira de faz-de-conta é marcante entre os conteúdos contrários e desconhecidos, nao interferindo na solução de silogismos envolvendo fatos conhecidos. 
TIPOS DE JUSTIFICATIVAS

Dois juizes independentes classificaram as justificativas com $95.3 \%$ de acordo. Os julgamentos discrepantes foram apresentados a um terceiro juiz, que trabalhou em 36 justificativas. Esta terceira avaliação foi tida como final.

\section{Justificativas Teóricas}

A Tabela 4 indica que no Grupo de Brincadeira foram oferecidas mais justificativas teóricas do que no Grupo Verbal, principalmente nos fatos contrários e nos fatos desconhecidos. Também fica evidente que as crianças brasileiras não escolarizadas ofereceram este tipo de justificativa em menor número, em todos os tipos de fatos e em ambos os grupos, do que as crianças inglesas e brasileiras escolarizadas. Nos problemas envolvendo Modus Ponens, o modo teórico foi apenas um pouco mais utilizado do que nos problemas envolvendo Modus Tollens.

A fim de checar estas afirmações, os dados foram submetidos a uma nova análise com Grupo (2), Tipos de Fato (3), Formas de Silogismo (2) e Amostra (3) como fatores e número de justificativas teóricas como variável dependente. Esta análise mostrou um efeito significativo para Grupo $(\mathrm{F}(1,108)=103.15, \mathrm{p}<.001)$, para Tipos de Fato $(\mathrm{F}(2,108)=15.38$, $\mathrm{p}<.001)$ e para Amostra $(\mathrm{F}(2,108)=46.04, \mathrm{p}<.001)$.

As médias de justificativas teóricas nas três amostras foram comparadas usando-se o teste de Newman-Keuls. A análise mostrou que as crianças inglesas ofereceram maior número dessas justificativas do que as crianças brasileiras não escolarizadas $(\mathrm{p}<.01)$ e escolarizadas $(\mathrm{p}<.05)$. Estas últimas ofereceram mais justificativas teóricas que as crianças não escolarizadas $(\mathrm{p}<.01)$.

Este teste também foi aplicado na análise das justificativas teóricas nos três tipos de fatos. Foram oferecidas mais justificativas teóricas aos fatos desconhecidos do que aos conhecidos $(\mathrm{p}<.01)$ e aos contrários $(\mathrm{p}<.05)$. A estes últimos houve maior número destas justificativas quando comparado aos conhecidos $(\mathrm{p}<.05)$. 
Tabela 4 - Número médio de Justificativas Teóricas, Empíricas e Arbitrárias em função de Grupo, Tipo de Fato e Forma de Silogismo nas três amostras

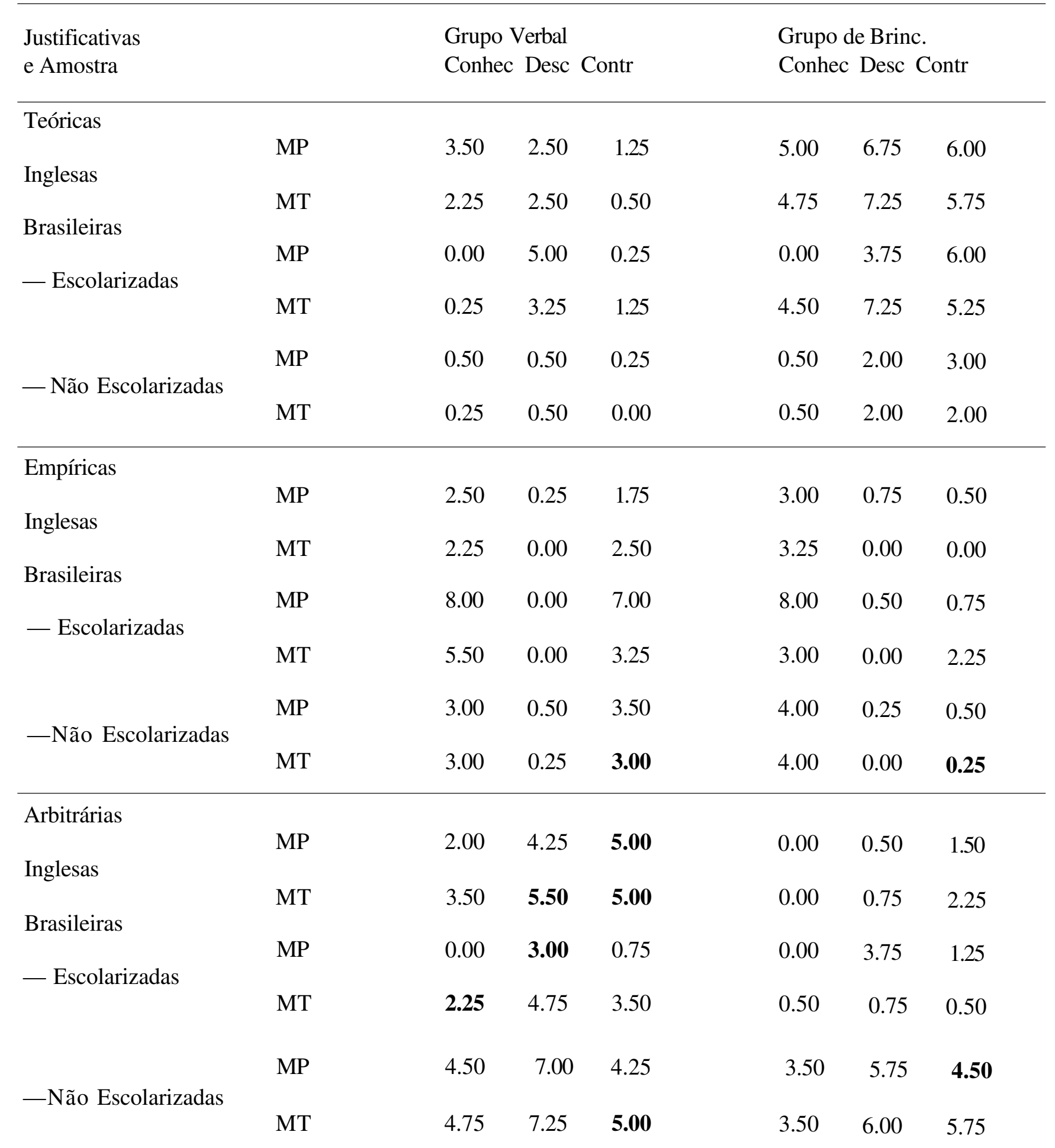


Pode-se ver na Tabela 4 que o número de justificativas empíricas foi maior no Grupo Verbal do que no Grupo de Brincadeira c, cm ambos grupos, foi mais utilizada nos fatos conhecidos do que nos outros dois tipos de fatos. As crianças brasileiras não escolarizadas ofereceram este tipo de justificativa mais freqüentemente do que as crianças inglesas e brasileiras escolarizadas, principalmente nos fatos conhecidos. $\mathrm{O}$ número de justificativas empíricas nos problemas envolvendo Modus Ponens é bastante similar ao oferecido àqueles envolvendo Modus Tollcns. A análise de variância com Grupo X Tipos de Fato X Formas de Silogismo X Amostra como fatores e justificativas empíricas como variável dependente confirma a existência de um efeito significativo para Grupo $(\mathrm{F}(1,108)=$ $14.08, \mathrm{p}<.001)$, Tipos de Fato $(\mathrm{F}(2,108)=100.32, \mathrm{p}<.001)$, Amostra $(\mathrm{F}(2,108)=22.65, \mathrm{p}<.001)$ e Formas de Silogismo $(\mathrm{F}(1,108)=9.08, \mathrm{p}<.01)$.

As médias de justificativas empíricas oferecidas por cada uma das amostras foram analisadas com o teste de Newman-Keuls. As crianças inglesas c brasileiras não escolarizadas não diferiram significativamente no número destas justificativas. No entanto, as duas amostras ofereceram menos explicações empíricas às suas respostas do que as brasileiras escolarizadas $(\mathrm{p}<.01)$.

Quanto aos Tipos de Fato, este teste mostra que maior número de explicações empíricas foi oferecido aos fatos conhecidos do que aos contrários $(\mathrm{p}<.01)$ e aos desconhecidos $(\mathrm{p}<.01)$. Também este tipo de justificativa foi mais utilizada para os fatos contrários quando comparados aos desconhecidos $(\mathrm{p}<.01)$.

\section{Justificativas Arbitrárias}

Ainda na Tabela 4 pode-se notar que as justificativas arbitrárias foram mais utilizadas no Grupo Verbal do que no Grupo de Brincadeira, principalmente nos fatos desconhecidos. As crianças brasileiras não 
escolarizadas ofereceram estas justificativas $\mathrm{cm}$ maior escala do que as crianças inglesas e as brasileiras escolarizadas. A análise de variância com Grupo X Tipos de Fato X Formas de Silogismo X Amostra como fatores e Justificativas Arbitrárias como variável dependente mostrou um efeito significativo para Grupo $(\mathrm{F}(1,108)=28.79, \mathrm{p}<.001)$, para Tipos de Fato $(\mathrm{F}(2,108)=13.50), \mathrm{p}<.001)$, e para Amostra $(\mathrm{F}(2,108)=39.74, \mathrm{p}<001)$.

As médias das justificativas arbitrárias nas três amostras foram comparadas usando-se o Teste de Newman-Keuls. Esta análise mostrou que as crianças brasileiras escolarizadas $\mathrm{c}$ inglesas ofereceram um número significativamente menor destas justificativas do que as brasileiras não escolarizadas $(\mathrm{p}<.01)$. Já as crianças brasileiras escolarizadas ainda ofereceram menor número destas justificativas do que as inglesas $(\mathrm{p}<.01)$.

Em relação aos três tipos de fatos, o número deste tipo de justificativa não diferiu entre os fatos contrários c os fatos desconhecidos. No entanto, as crianças ofereceram um número menor de justificativas arbitrárias para os fatos conhecidos e para os fatos contrários quando comparado com os fatos desconhecidos $(\mathrm{p}<.01)$.

DISCUSSÃO

O principal objetivo deste estudo foi investigar o efeito dos fatores contexto e escolarização na resolução de problemas de tipo silogístico. Os resultados indicam claramente a importância do contexto na resolução deste tipo de problemas. Este resultado confirma uma série de outros estudos sobre esta relação. De fato, como nos estudos de Dias e Harris (1988a, 1988b e 1990), quando os problemas foram apresentados em um contexto de brincadeira de faz-de-conta, o desempenho das crianças, independentemente se brasileiras ou inglesas, escolarizadas ou não, foi bom em ambas as formas de silogismo, nos três tipos de fatos.

Este bom desempenho diminuiu bastante quando o modo verbal comum de apresentação dos problemas era utilizado. Isto ocorreu principalmente quando o conteúdo dos problemas era desconhecido ou 
contrário às experiências do dia-a-dia. No entanto, o bom desempenho das crianças de ambos os grupos não sofreu alteração quando o conteúdo dos problemas era conhecido. Este fenômeno foi também encontrado em estudos anteriores com crianças inglesas (ver Dias e Harris, 1988a, 1988b e 1990). A explicação sugerida por esses autores 6 a de que as respostas corretas para os problemas com fatos conhecidos são conduzidas tanto pelo modo dedutivo, refletido nas justificativas teóricas, quanto pelo modo empírico, refletido nas justificativas empíricas. No entanto, somente o modo dedutivo conduz às respostas corretas, quando os fatos são desconhecidos ou contrários. Assim, as crianças do Grupo Verbal foram menos capazes $\mathrm{cm}$ adotar uma atitude teórica nos problemas envolvendo estes tipos de conteúdos, porém conseguiram um bom desempenho quando os fatos eram conhecidos.

Quanto às duas formas de inferência válida, tanto as crianças inglesas quanto as brasileiras obtiveram um melhor desempenho nos problemas envolvendo Modus Ponens do que naqueles envolvendo Modus Tollens, replicando os resultados alcançados por Ennis (1976), Kadroff e Roberge (1975), Evans e Lynch (1973), Dias e Harris (1988b).

Neste estudo procurou-se testar o pressuposto de que a escolarização seria um pré-requisito para o raciocínio dedutivo. Diferentemente das crianças inglesas e brasileiras escolarizadas, as crianças não escolarizadas não tinham tido qualquer experiência escolar c, apesar de os resultados terem mostrado que estas últimas obtiveram um desempenho mais fraco do que as crianças escolarizadas, a diferença não foi tão forte como aquela encontrada por Scribner (1977). No estudo de Scribner, as crianças não escolarizadas alcançaram resultados no "nível de chance", adotando o viés empírico. Já no presente estudo, as crianças não escolarizadas, embora tenham com frequiência recorrido às justificativas arbitrárias e empíricas, conseguiram adotar uma atitude teórica quando a condição de faz-de-conta foi utilizada, particularmente para os fatos desconhecidos e para os fatos contrários às suas experiências diárias. 
Assim, apesar de os estudos transculturais enfatizarem a importância da escolarização no desenvolvimento de uma atitude teórica, nossos resultados mostram que a escolarização pode, de fato, provocar tal atitude, porém não a engendra em primeiro lugar. Outros fatores podem estar envolvidos no uso do raciocínio lógico dedutivo como nossos dados mostram.

Além da diferença entre crianças escolarizadas (inglesas c brasileiras) e crianças brasileiras não escolarizadas no tipo de justificativa em função do contexto da situação de teste, é interessante notar a diferença entre os dois grupos de crianças (escolarizadas x não escolarizadas) em relação ao tipo de justificativaperse. As crianças brasileiras não escolarizadas deram um número maior de justificativas empíricas e arbitrárias enquanto as crianças escolarizadas inglesas e brasileiras ofereceram maior número de justificativas teóricas. De fato, ambas as amostras de crianças escolarizadas, apresentaram um maior número de justificativas teóricas, seguidas pelas empíricas e, por último, pelas as arbitrárias. A seqüência de justificativas das crianças não escolarizadas foi: arbitrárias, empíricas c teóricas. Por que esta diferença $\mathrm{cm}$ termos de justificativas? Uma análise da maneira como a escola procede no seu processo de ensino permite uma melhor compreensão dessa diferença.

$\mathrm{Na}$ forma como a instituição escola é estruturada e o conhecimento transmitido, há um privilégio da dimensão abstrata e simbólica cm relação à dimensão empírica e concreta. Enquanto as crianças, no contexto informal da vida diária, aprendem através de observação e participação direta, onde o que é aprendido será em seguida colocado em prática, na sala de aula o conhecimento é transmitido principalmente de forma oral, sendo limitada a participação ativa e direta. Não só conceitos abstratos, mas também conceitos concretos são ensinados através de dimensões abstraídas da experiência concreta (por exemplo, o uso de diagramas, livros, modelos, palavras, símbolos). Muitas vezes conceitos ou eventos são estudados, discutidos ou até desenhados sem nunca se promover e haver uma experiência direta. Em outras palavras, tende-se a privilegiar a generalização através da eliminação do conteúdo concreto, a fim de se alcançar um alto nível de abstração. Isto é, tende-se a enfatizar a 
compreensão de estruturas lógicas independentemente de seus conteúdos; tende-se a enfatizar a diferenciação de forma c conteúdo como altamente significativa; tende-se a separar o objeto de ensino do contexto normal $\mathrm{cm}$ que este é praticado. Esta separação permite transformações $\mathrm{cm}$ formas simbólicas. Desta forma, a escola não só privilegia um certo tipo de atividade como também a aborda de forma particular.

Esta ênfase da escola no uso preferencial de elementos teóricoabstratos explica em parte porque em nosso estudo as crianças escolarizadas ofereceram maior número de justificativas teóricas do que empíricas ou arbitrárias, $\mathrm{cm}$ relação ao grupo de crianças não escolarizadas. A escola, por sua natureza, tende a melhorar a capacidade de raciocínio descontextualizado. O raciocínio lógico implícito nos problemas do nosso estudo é um claro exemplo de raciocínio descontextualizado, cuja correta solução esta baseada no uso de estratégias mentais que não possuem nenhuma relação com o significado dos seus enunciados na vida concreta.

\section{Conclusão}

Apesar de os dados apontarem para um melhor desempenho das crianças escolarizadas cm relação às não escolarizadas, estas últimas, em comparação com estudos anteriores (ver Scribner, 1977), alcançaram um nivel de desempenho muito superior, raciocinando teoricamente quando a condição de faz-de-conta era apresentada. Desta forma, estes dados nos indicam que, apesar de a experiência escolar ser importante e não subestimávcl (Laboratory of Human Cognition, 1983; Luria, 1976;Neisser, 1976; Olson, 1977), no entanto, não é a única experiência capaz de afetar o desenvolvimento cognitivo, além da importância do contexto experimental $\mathrm{cm}$ uma situação de exame.

O problema do contexto e da experiência do sujeito, mais uma vez, volta a mostrar sua importância na situação de teste (por exemplo, Ceei e Liker, 1986a e 1986b; Ceei e Bronfenbrenner, 1985; Ceei e Roazzi, 199; Roazzi, 1989; Roazzi, Bryant e Schliemann, 1988; Roazzi c Bryant, 
199-; Dias e Harris, 1988a, 1988b, 1989 e 1990). De fato, já foi demonstrado na literatura que muitos testes cognitivos elaborados por pesquisadores ocidentais não são geralmente apropriados para populações tradicionais (Cole, Gay, Glick, Sharp, 1971). Além do contexto de exame, a falta de familiaridade com o material (Roazzi, 1986a e 1986b; Serpell, 1971a e 1971b) e até a falta de motivação (Roazzi c Dias, 199-) podem tornar particularmente difícil a inferência de níveis de competência a partir do nivel de desempenho. Nossos dados apontam na mesma direção. É possível assim hipotetizar a ocorrência de melhores níveis de desempenho de sujeitos não escolarizados $\mathrm{cm}$ tarefas cognitivas, uma vez que seja considerada com mais atenção e cuidado a variável contexto de exame do sujeito.

De toda maneira, o efeito da variável contexto e da experiência do sujeito com o tipo de problema apresentado pode ser também encontradas em sujeitos de NSE médio com um grau de instrução universitária. De fato, esta desvantagem $\mathrm{cm}$ saber lidar com problemas silogísticos não é somente uma prerrogativa de indivíduos não escolarizados; também indivíduos com um nivel de instrução universitária podem apresentar dificuldades quando silogismos são apresentados, não cm uma forma escolar, como eles estão geralmente acostumados a lidar. Este resultado interessante foi encontrado por Henle (1962) em um estudo em que silogismos eram apresentados a uma pequena amostra de estudantes universitários, de uma forma que facilmente poderia ser encontrada no dia-a-dia das pessoas. $\mathrm{O}$ objetivo do estudo era sublinhar a necessidade de se considerar o papel das crenças do sujeito no raciocínio, isto é, como o sujeito interpreta os objetivos e os materiais da tarefa lógica ${ }^{1}$. Um tipo de silogismo utilizado por Henle foi apresentado da seguinte forma:

\footnotetext{
'O papel desempenhado pelas crenças no raciocínio lógico é um tópico baslante controvertido na psicologia cogniliva. Inúmeros estudos lêm mostrado que os sujeitos silo influenciados pelas suas crenças, quando avaliam se determinadas conclusões derivam de premissas silogisticas(Wilkins, 1928; Janis cl-rincli, 1943; Morgan e Moinou, 1944; 1.cllbrd, 1946; I lenlc e Michael, 1956; Thouless, 1959; Fcathcr, 1964; Kaulman c Goldslein, 1967; Oakhill, Cambam e Johnson-laird, 1990). Ao mesmo tempo, um outro número de pesquisadores afirmam que a partir do momento em que os príncipios dedutivos estão baseados em regras de tipo lógico, estes príncipios nao podem ser influenciados por crenças (Falmagne, 1975; Revlin e Leirer, 1978; Kevlin. Lcirer, Yopp e Yopp, 1980; Evans, Barston e Pollard, 1983). Apesar de existirem até o momento mais evidências em favor do primeiro grupo de pesquisadores, ainda várias questões permanecem sem solução, como por exemplo: em que situações as crenças possuem seus efeitos? Como a crença interage com o nivel de dificuldade do problema? etc.
} 
Um grupo de mulheres estava discutindo seus problemas de casa quando uma delas disse:

- É muito importante estar discutindo o que se passa em nossas mentes.

- Passamos tanto tempo na cozinha que os problemas de casa estão em nossas mentes.

- Então, é importante estar discutindo-os.

Em seguida, pedia-se aos sujeitos para avaliarem a validade dos argumentos e justificarem a resposta. Da mesma forma como fizeram os sujeitos da tribo Kpelle de Cole, Gay, Glick e Sharp (1971) e os camponeses do Ubequistão de Luria (1976), um número considerável de estudantes universitários recusava-se a responder e nao aceitava resolver o problema como um problema de tipo lógico ${ }^{2}$. Os sujeitos eram incapazes de distinguir a validade lógica da verdade dos fatos ${ }^{3}$.

Se o contexto e a forma de apresentação de um problema são fatores importantes para indivíduos com um alto nivel de instrução na resolução de problemas silogísticos, mais importantes serão estas variáveis para os indivíduos não escolarizados? A criança não escolarizada pode não estar acostumada ao tipo de procedimento experimental utilizado na situação de teste ou até não estar motivada o suficiente para responder naquele contexto de exame. Afinal, não é sem sentido esperarmos que uma pessoa que nunca foi para escola e que, conseqüentemente, nunca vivenciou situações ou questões de tipo escolar, perceba que o problema silogístico

\footnotetext{
-O Fato de um individuo estar cm um curso universitário nao significa necessariamente que este utiliza na resolução de problemas um tipo de raciocinio lógico. Existem dados da literatura que apontam uma dificuldade notável por pane de estudantes universitárias nao treinados cm lógica cm raciocinar de acordo com os parâmetros próprios da lógica. Para Dominowski (1977). por exemplo, essa porcentagem em raciocinar logicamente nao chegava a $10 \%$.

${ }^{\mathrm{J}}$ Por exemplo, respostas de recusa em raciocinar cm termos de silogismo: "Nao $i$ importante (alar sobre coisas que passam em nossas mentes, a não ser que elas nos aborreçam", ou: "Somente porque uma mulher passa a maioria do seu tempo na cozinha nao significa necessariamente que ela pense somente em problemas de casa". É como se os sujeitos avaliassem as conclusões dos silogismos cm relação a suas crenças, mais do que através de uma análise lógica. Eni relação a estes resultados, Henle (1962, p.3 75) afirma que: "Sc considerarmos o material e a tarefa da forma como eles foram realmente compreendidos pelos indivíduos, é dificil encontramos evidencias de raciocínio errado. Deve-se concluir que a presença de erros nao se constitui uma evidência que as leis da lógica São irrelevantes para o pensamento real". Desta forma, para Henle, as pessoas raciocinam, na maioria das vêzes, de forma racional, mas freqüentemente nao interpretam corretamente as premissas ou a solicitação do experimentador, de forma a incorrer em erros. Conseqüentemente, para Henle, considerar as possiveis interpretações da tarefa por pine do sujeito $\mathrm{cm}$ exame pode tomar, com certeza, o comportamento e desempenho do mesmo mais compreensíveis. Estes resultados São consistentes com os resultados obtidos por Ceraso e Provitera (1971), os quais tem mostrado como premissas claras c sem ambigüidades levam as pessoas a cometerem muito menos erros do que premissas de tipo tradicional contendo mais ambigüidades.
} 
que o experimentador lhe apresenta é um quebra-cabeça lógico, um tipo de jogo no qual ele como sujeito deve abstrair-se do significado que as primeiras duas frases contêm e prestar atenção só às palavras ou ao significado literal que estas apresentam. Esta habilidade de se abstrair do significado e prestar atenção somente à forma do problema apresentado não é necessariamente uma habilidade cognitiva, mas uma habilidade em saber lidar com convenções sociais baseadas na experiência sociocultural do sujeito - o que poderia ser chamado de "curriculum oculto" cuja responsabilidade, $\mathrm{cm}$ termos de transmissão, é principalmente atribuída à escola.

O mesmo não acontece com as crianças escolarizadas que possuem experiências de situações semelhantes dentro da sala de aula. Estas crianças, ao aprenderem a ler, a escrever e a fazer contas, além de adquirirem um novo sistema de representação, devido à natureza abstrata de nosso sistema alfabético para a representação dá linguagem, como também à natureza abstrata do nosso sistema numérico decimal, absorvem toda uma série de atitudes e valores que lhe facilitam a forma de enfrentar a solução de problemas de tipo escolar. Estas crianças apresentam um desejo de autonomia, uma menor preocupação com as figuras de autoridades tradicionais, e uma vontade de obter prazer pelos desafios implícitos nos problemas que se lhe apresentam sem serem contingentes a recompensas concretas externas e, enfim, às vêzes, uma abertura para novas perspectivas e formas de solucionar problemas.

Para concluir, talvez as diferenças entre sujeitos escolarizados e não escolarizados possam variar não só em função do contexto da situação de exame e do nível de escolarização, mas também em função de uma série de outras variáveis como as sugeridas acima, variáveis estas, importantes, mas que, de toda maneira, não possuem relação alguma com habilidades intelectuais. Neste sentido, torna-se necessária a realização de pesquisas nesta área, que considerem esses outros fatores citados, para uma melhor compreensão do papel do contexto e da escolarização no desenvolvimento cognitivo da criança. 


\section{Referencias bibliográficas}

BRAINE, M.D.S., Rumain, B. Logical reasoning. In: FLAVELL, J.H., MARKMAN, E.M. (Eds.). Cognitive development: Carmichael's manual of child psychology. 4.ed. New York: John Wiley, 1983. v.3.

CECI, S.J., LIKER, J. A day at lhe races: a study of IQ, expertise, and cognitive complexity. Journal of Experimental Psychology: General, n. 115, p.255-266, 1986a.

Academic and nonacademic intelligence: an experimental separation. In: STERNBERG, R.J., WAGNER, R. (Eds.). Practical intelligence: origins of competence in the everyday world. New York: Cambridge University Press, 1986b.

CECI, S.J., BROFENBRENNER, U. Don't forget to lake the cupcakes out of the oven: strategic time-monitoring, prospective memory and context. Child Development, n.56, p. 175-190, 1985.

CECI, S.J., ROAZZI, A. The effects of context on cognition: portraits from Brazil. In: STERNBERG, R. (Ed.). Mind in context: mteractionist perspectives on human intelligence. Cambridge: Cambridge University Press, 1994.

CERASO, J., PROVITERA, A. Sources of error in syllogistic reasoning. Cognitive Psychology, n.l, p.400-410, 1971.

COLE, M., GAY, J., GLICK, J., SHARP, D.W. The cultural context of learning and thinking. New York: Basic, 1971.

COLE, M., SCRIBNER, S. Culture \& thought: a psychological introduction. New York: John Wiley, 1974.

DIAS, M.G.B.B. Da lógica do analfabeto à lógica do adolescente: há progresso? Arquivos Brasileiros de Psicologia, Rio de Janeiro, v.39, p.29-40, 1987.

DIAS, M.G.B.B., HARRIS, PL. The effect of make-believe play on deductive reasoning. British Journal of Developmental Psychology, v.6, p.207-221,1988a.

. Reality versus imagination: their effect on deductive reasoning. [S.1.], 1988b. Trabalho apresentado na Annual Conference 1988 of the British Psychological Society - Developmental Psychology Section, Pais de Gales, set. 1988. 
. O efeito da brincadeira de faz-de-conta no raciocínio da criança. Arquivos Brasileiros de Psicologia, Rio de Janeiro, v.41, n.2, p.95-105, 1989.

. The influence of the imagination on reasoning by young children. British Journal of Developmental Psychology, v.8, p.305-318, 1990.

DOMINOWSKI, R.L. Reasoning. Interamerican Journal of Psychology, n.1 1, p.68-77, 1977.

ENNIS, R.H. An alternative to Piaget's conceptualization of logical competence. Child Development, n.47, p.903-919, 1976.

EVANS, J.St.B.T., BARSTON, I, POLLARD, P. On the conflict between logic and belief in syllogistic reasoning. Memory and Cognition, v.l 1, p.295306, 1983.

EVANS, J.St.B.T., LYNCH, J.S. Matching bias in the selection task. British Journal of Psychology, v.64, p.392-397, 1973.

FALMAGNE, R.J. Reasoning: representation and process. Hillsdale: Lawrence Erlbaum Associates, 1975.

FEATHER, N.T. Acceptance and rejection of arguments in relation to attitude strength, critical ability and intolerance of inconsistency. Journal of $A b$ normal and Social Psychology, v.69, p. 127-136, 1964.

HAWKINS, J., PEA, R.D., GLICK, J., SCRIBNER, S. Merds that laugh don't like mushrooms: evidence for deductive reasoning by preschoolers. Developmental Psychology, V.20, n.4, p.584-594, 1984.

HENLE, M. On the relation between logic and thinking. Psychological Review, v.69, p.366-378, 1962.

HENLE, M., MICHAEL, M. The influence of attitudes on syllogistic reasoning. Journal of Social Psychology, v.44, p. 115-127, 1956.

HOLANDA, V.M.L. A influência do contexto de faz-de-conta no exame de problemas silogísticos deformas válidas e inválidas por crianças de 4 e 6 anos de idade. Recife, 1989. Dissertação (Mestrado cm Psicologia) UFPE. 
JANIS, I., FRINCH, P. The relationship between attitudes towards conclusions and errors in judging logical validity of syllogisms. Journal of Experimental Psychology, v.33, p.73-77, 1943.

KADROFF, J., ROBERGE, J. Developmental analyses of the conditional reasoning abilities of primary-grade children. Developmental Psychology, v. 11, p.21-28, 1975.

KAUFMAN, H., GOLDSTEIN, S. The effects of emotional value of conclusions upon distortion in syllogistic reasoning. Psychonomic Science, v.7, p.367-368, 1967.

LABORATORY OF COMPARATIVE HUMAN COGNITION. Culture and cognitive development. In: MUSSEN, PII. (Ed.). Handbook of child psychology. New York: Wiley, 1983. v.l: History, theory and Methods.

LEFFORD, A. The influence of emotional subject matter on logical reasoning. Journal of General Psychology, v.34, p. 127-151,1946.

LURIA, A.R. Cognitive development; its cultural and social foundations. Cambridge: Harvard University Press, 1976.

MORGAN, J., MORTON, J. The distortion of syllogistic reasoning produced by personal convictions. Journal of Social Psychology, v.20, p.39-59, 1944.

NEISSER, U. Cognition and reality. San Francisco: W.H. Freeman, 1976.

OAKHILL, J., GARNHAM, A., JOHNSON-LAIRD, PN. Belief bias effects in syllogistic reasoning. In: G1LHOOLY, K.J., KEANE, M.T.G., LOGIE, R.H., ERDOS, G. (Eds.). Lines of thinking: reflections on the psychology of thought. New York: John Wiley, 1990. v.l : Representation, reasoning, analogy and decision making.

OLSON, D.R. From utterance to text: the bias of language in speech and writing. Harvard Educational Review, n.47, p.257-281, 1977.

REVLIN, R., LEIRER, V. The effect of personal biases on syllogistic reasoning: rational decisions from personalized representations. In: RFVL1N, R., MAYER, R.E. (Eds.). Human reasoning. Washington, DC: Winston-Wiley, 1978. 
REVLIN, R., LEIRER, V., YOPP, H., YOPP, R. The belief-bias effect in formal reasoning: the influence of knowledge on logic. Memory and Cognition, v.8, p.584-592, 1980.

ROAZZI, A. Implicações metodológicas na pesquisa transcultural: a influência do contexto social cm tarefas lógicas. Arquivos Brasileiros cie Psicologia, V.38, n.3, p.71-91, 1986a.

. Social context in experimental psychology. Ricerche eli Psicologia, VÃ1T23-45, $1986 b$.

. Desenvolvimento cognitivo e pesquisa piagetiana. [S.1.], 1989. Trabalho apresentado no II Simpósio Brasileiro de Pesquisa c Intercâmbio Científico, Gramado, RS, 19 a 23 de abr. 1989.

ROAZZI, A., BRYANT, P.E.B. Social class, context and cognitive development. In: LIGHT, P, BUTTERWORT, G. (Eds.). Context anel cognition: ways of learning and knowing. Herts: Harvester \& Wheatshcaf, 1991.

ROAZZI, A., BRYANT, P.E.B., SCHLIEMANN, A.D. Context effects on children's performance of conservation task. [S.1.], 1988. Trabalho apresentado na Annual Conference of the British Psychological Society, Developmental Psychology Section, set. 1988.

ROAZZI, A., DIAS, M.G. A influência da motivação em tarefas cognitivas. Psicologia: Teoria e Pesquisa, [199-].

SANTOS, C.M.M. Fazendo de conta tudo pode. Recife, 1989. Dissertação (Mestrado em Psicologia) - UFPE.

SCRIBNER, S. Recall of classic sillogisms: a cross-cultural investignation of erros in logical problems. In: FALMAGNE, R. (Ed.) Reasoning representation and process. Hilldalc: Erlbaum, 1975.

Models of thinking and ways of speaking: culture and logic reconsidered. In: JOHNSON-LAIRD, PN., WASON, PC. (Eds.). Thinking. New York: Cambridge University Press, 1977. p.483-500.

SERPELL, R. Discrimination of orientation by Zambian children. Journal of Comparative and Psychological Psycology, n.75, p.312-316, 1971 a. 
. Preference for specific orientation of abstract shapes among Zambian children. Journal ofCross-Cultural Psychology, v.2, p.225-239, 1971 b.

THOULESS, R. A. Effect of prejudice on reasoning. British Journal ofPsychology, V.50, p.289-293, 1959.

WILKINS, M. The effect of changed material on ability to do formal syllogistic reasoning. Archives of Psychology, v. 16, p. 102, 1928.

Recebido cm 31 de maio de 1994

Maria da Graça B.B. Dias c Antonio Roazzi são professores-adjuntos do mestrado em Psicologia da Universidade Federal de Pernambuco (UFPE).

The main aim of this study was to investigate the effect of makebelieve mode, form of syllogisms and type of fact in 5-year-old schooled children from medium SES families in England and in nonliterate 5year-old unschooled children from low SES families in Brazil. This study was a test of the claim that schooling is a pre requesite for deductive reasoning. The results showed that children, English or Brazilian, produced more correct responses and theoretical justifications in the makebelieve play condition than in the standard verbal mode. This was especially true for unknown facts and contrary facts. This pattern held for both form forms of syllogism, although in general children 's performance on Modus Ponens inferences was more accurate than on Modus Tollens inferences. Unschooled children s performance was poorer than schooled children s performance. However, this difference was not so strong as that found in the studies reported by Scribner (1977) where unschooled children performed at chance level, adopting the empirical bias. In this study, the unschooled children, although they very often appealed to arbitratyjustifications, could adopt a theoretical attitude when the makebelieve mode was used. 
Ce travail de recherche a eu comme objectif principal l'investigation de <'effet du mode faire-semblant, des formes de syllogisme et des types défaits sur la réussite d'enfants anglais de cinq ans, scolarisés, niveau socio-économique moyen, et d'enfants brésiliens de même âge, nonscolarisés et de bas niveau socio-économique. Les résultats indiquent aue les enfants, scolarisés ou non, ont produit un plus grand nombre de réponses correctes, ainsi que de justificatives théoriques dans la condiction experimentale faire-semblant, par rapport à la condiction verbale commune. Cet effet a été plus net en ce qui concerne des faits nconnus et des faits contraires aux expériences des enfants. Ce résultat a été observé pour es deux formes de syllogisme étudiées, quoi que, en général, le nombre de réponses correctes a été plus important chez les enfants travaillant avec des problèmes em Modus Ponens, par raport à ceux travaillant sous modus Tollens. Les enfants non-scolarisés ont obtenu des scores plus bas que ceux des enfants scolarisés. Néanmoins, cette différence $n$ 'a pas été aussi forte que celle mentionnée par Scribner (1977), qui a pu constater des scores bas chez des enfants non-scolarisés adoptant un biais empirique. Dans notre étude, bien que les enfants nonscolarisés aient fréquemment fait appel à des justifications arbitraires, ils ont, d'autre part, eu recours à une attitude théorique quand la condiction faire-semblant a été proposée.

El objetivo principal de este estudio fue investigar el efecto del modo de fingir formas de silogismos y tipos de hechos en el rendimiento de niños ingleses de 5 anos de edad, escolarizadas, de NSE médio y de linos brasileños de 5 anos de edad, sin escolaridad de NSE bajo. Los resulados mostraron que os niños, independientemente de si eran escolarizados o no, producían mayor número de respuestas correctas $e$ mayor número de justificativas teóricas en la condición del juego de fingir que en la condición verbal común. Este efecto fue especialmente 
marcante para los hechos desconocidos y para los hechos contrários a Ias experiencias de los ninos. Este padrón fue conservado para ambas formas de silogismos, no obstante, en general el rendimiento de los ninos en los problemas envolviendo Modus Ponens haya sido mas preciso que en los problemas envolviendo Modus Tollens. Los ninos sin escolaridad obtuvieron resultados menores que los escolarizados. Sin embargo, esta diferencia no fue tan fuerte como aquella relatada por Scribner (1977) donde los ninos sin escolaridad obtuvieron bajos resultados adoptando una inclinación empírica. En nuestro estúdio, a pesar de los ninos sin escolaridad haber recurrido frecuentemente a justificativas arbitrarias, estos adoptaron una actitud teórica cuando fue usada Ia condición de fingir. 